\title{
Pyruvate dehydrogenase complex stimulation promotes immunometabolic homeostasis and sepsis survival
}

\author{
Charles E. McCall, ${ }^{1}$ Manal Zabalawi, ${ }^{1}$ Tiefu Liu, ${ }^{1}$ Ayana Martin, ${ }^{1}$ David L. Long, ${ }^{1}$ Nancy L. Buechler, ${ }^{2}$ \\ Rob J. W. Arts, ${ }^{3}$ Mihai Netea, ${ }^{3}$ Barbara K. Yoza, ${ }^{4}$ Peter W. Stacpoole, ${ }^{5}$ and Vidula Vachharajani ${ }^{1,2}$ \\ 'Department of Internal Medicine/Molecular Medicine and 'Department of Anesthesiology, Wake Forest School of \\ Medicine, Winston-Salem, North Carolina, USA. ${ }^{3}$ Department in Internal Medicine and Radboud Center for Infectious \\ Diseases, Radboud Medical Center, Nijmegen, Netherlands. ${ }^{4}$ Department of Surgery/General Surgery and Trauma, Wake \\ Forest Medical School, Winston- Salem, North Carolina, USA. ${ }^{5}$ Department of Medicine, Division of Endocrinology, \\ Diabetes \& Metabolism, and Department of Biochemistry and Molecular Biology, University of Florida College of Medicine, \\ Gainesville, Florida, USA.
}

Limited understanding of the mechanisms responsible for life-threatening organ and immune failure hampers scientists' ability to design sepsis treatments. Pyruvate dehydrogenase kinase 1 (PDK1) is persistently expressed in immune-tolerant monocytes of septic mice and humans and deactivates mitochondrial pyruvate dehydrogenase complex (PDC), the gate-keeping enzyme for glucose oxidation. Here, we show that targeting PDK with its prototypic inhibitor dichloroacetate (DCA) reactivates PDC; increases mitochondrial oxidative bioenergetics in isolated hepatocytes and splenocytes; promotes vascular, immune, and organ homeostasis; accelerates bacterial clearance; and increases survival. These results indicate that the PDC/PDK axis is a druggable mitochondrial target for promoting immunometabolic and organ homeostasis during sepsis.

Conflict of interest: The authors have declared that no conflict of interest exists.

Submitted: December 15, 2017

Accepted: June 21, 2018

Published: August 9, 2018

\section{Reference information:}

JCI Insight. 2018;3(15):e99292.

https://doi.org/10.1172/jci.

insight.99292.

\section{Introduction}

The nutrient substrates that cells consume for energy determines their responses to infection (1). Increases in glucose uptake and glycogen breakdown used in glycolysis and glucose oxidation support the anabolic differentiation, replication, and resistance effector state of innate and adaptive immune cells $(2,3)$. In contrast, fatty acid oxidation supports immune repressor cells, memory, and immune tolerance in monocytes and T cells (4). However, the extreme systemic stress of sepsis, and the associated anabolic and catabolic energy imbalance, provokes a pseudo-starvation-like state that resembles the metabolic torpor of hibernation, frequently resulting in a lethal state of mitochondrial energy failure $(5,6)$. This breach between deprived glucose substrate for high energy demand and the low energy supply limits immune resistance and organ function and regeneration. Currently, there are no molecular-based treatments available that counter this life-threatening energy crisis.

We previously reported that $\mathrm{NAD}^{+}$-dependent nuclear sirtuin 1 (SIRT1) and SIRT6 epigenetically shift substrate selection in septic mouse or human monocytes from glucose fueling of immune resistance to fatty acid fueling of immune tolerance (7). During this metabolic switch, SIRT1 also increases mitochondrial SIRT3 expression, which supports mitochondrial catabolic energetics and activates antioxidant pathways (8). We also discovered that pharmacologically targeting tolerant septic mouse monocytes with a selective SIRT1 inhibitor reinstates glucose oxidation, reverses immune tolerance in microvasculature, reconstitutes the monocyte immune competence, and increases survival (9). Consistent with a mechanistic link between host energy imbalance and poor sepsis outcome, blood monocytes from septic humans and mice have broad defects in mitochondrial bioenergetics that support a clinical concept of immunometabolic paralysis (10). In that investigation, IFN- $\gamma$ administered to monocytes ex vivo and to humans in vivo partially corrected suppressed glycolysis and enhanced proimmune cytokine production in response to ex vivo TLR4 stimulation. Other reports also implicate dysregulated fatty acid, amino acid, and mitochondrial metabolism as risk factors for death in humans with septic shock and in nonhuman primate models of septic shock $(11,12)$. 
A

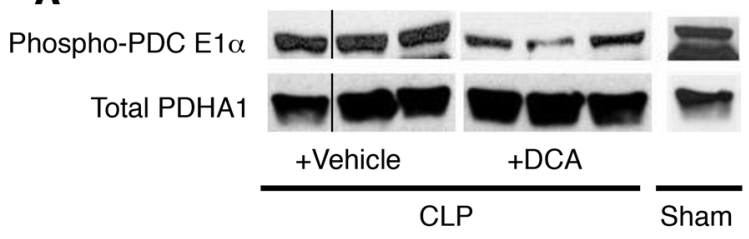

B

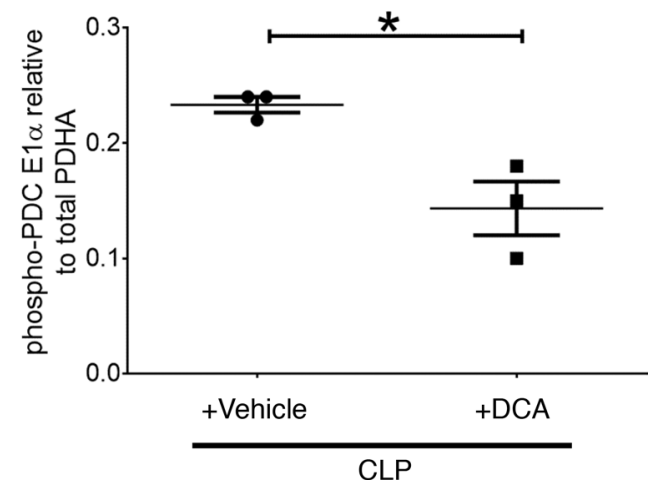

Figure 1. Dichloroacetate (DCA) treatment activates pyruvate dehydrogenase complex (PDC). (A and B) DCA represses phosphorylation at deactivating serine sites on PDC. Homogenized liver tissue obtained from SHAM, cecal ligation and puncture (CLP)+ DCA, and CLP+ vehicle was probed with a mixture of anti-phospho-S232 and S300 antibodies to PDC E1 $\alpha$ rate-limiting component for acetyl Co-A generation using Western blot. Anti-phospho-S232 and S300 on PDC in the CLP+ DCA group were significantly reduced vs. CLP+ vehicle. Western blot signals were quantified using Image). Unpaired $t$ test determined significance. ${ }^{*} P<0.05$.

Using the cecal ligation and puncture (CLP) model of murine sepsis (9), this study provides mechanistic insight into immunometabolic reprogramming in septic mice and introduces a potentially novel therapeutic approach to resolve sepsis inflammation by directly controlling mitochondrial substrate selection. The rationale for this work was based on our previous findings that human and mouse sepsis monocytes express and activate Pyruvate dehydrogenase kinase 1 (PDK1) (7), which inhibits the pyruvate dehydrogenase complex (PDC) by reversible phosphorylation of any 1 of 3 serine residues on its E1 $\alpha$ subunit (13). PDC E1 irreversibly decarboxylates pyruvate to acetyl coenzyme A (acetyl CoA), thereby functioning as a gate-keeper enzyme that links cytoplasmic glycolysis to the mitochondrial tricarboxylic acid (TCA) cycle and oxidative phosphorylation (OXPHOS) (14). Here, we show that inhibiting PDK with the pyruvate analog dichloroacetate (DCA) in septic mice restores PDC activity and improves mitochondrial respiration and cell bioenergetics. DCA stabilizes hemodynamics and reverses microvascular endotoxin tolerance, rebalances innate and adaptive immunity, and accelerates clearance of infecting organisms. As a consequence of these actions, DCA rehabilitates immunometabolism and organ function in septic mice and significantly improves survival.

\section{Results}

DCA activates PDC and promotes mitochondrial respiration and cell bioenergetics in septic hepatocytes and splenocytes. We previously found that PDK inactivates PDC to limit glucose carbon fueling of mitochondrial anabolic energy in human and mouse sepsis (7). Therefore, we determined whether i.p. administration of DCA 24 hours after CLP-induced sepsis can activate PDC in liver tissue samples assessed 6 hours after drug exposure (30 hours after CLP). We chose a drug dose of $25 \mathrm{mg} / \mathrm{kg}$ body weight, based on oral and parenteral dose administered clinically in literature (15). Figure 1, A and B, show significantly reduced phosphorylation of PDC E1 $\alpha$ serine residues S232 and S300, changes consistent with PDC reactivation (16). DCA did not alter total PDC protein levels.

We then determined the effects of PDK inhibition on mitochondrial respiration in isolated hepatocytes and splenocytes 6 hours after DCA treatment (as stated above). By stimulating PDC, DCA decreases lactate production from glycolysis and fuels the TCA cycle to foster OXPHOS (17). We employed Seahorse respirator technology and the mitochondrial stress test (Agilent) to assess DCA's impact on mitochondria. The Seahorse mitochondrial stress test simultaneously measures the oxygen consumption rate (OCR) and the extracellular acidification rate (ECAR) - the latter as an indirect measure of glycolysis and lactate secretion. OCR estimates ATP production by blocking Complex V with oligomycin, determines spare respiratory capacity (SRC) by using an uncoupling agent carbonyl cyanide-p-trifluoromethoxyphenylhydrazone (FCCP), and measures total mitochondrial respiration by Complex I-IV with rotenone and antimycin (18). We generated a total cell energy index plot, calculated by plotting the ratio 


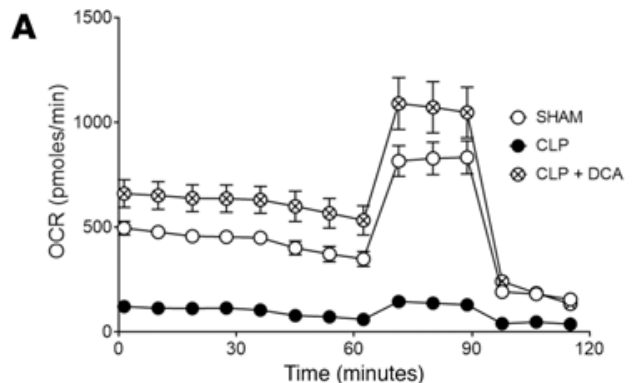

B
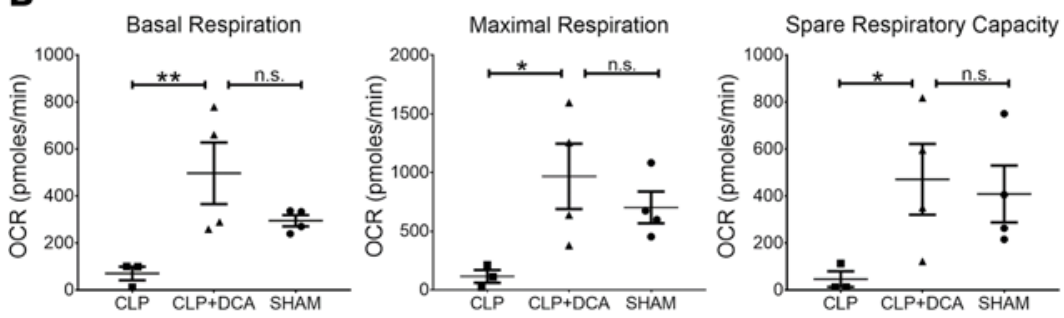

\begin{tabular}{|rr|c|c|}
\hline & $\begin{array}{c}\text { ECAR }(\mathrm{mpH} / \mathrm{min}) \\
\pm \text { SEM }\end{array}$ & $\begin{array}{c}\text { OCR }(\mathrm{pmoles} / \mathrm{min}) \\
\pm \text { SEM }\end{array}$ \\
\hline $\mathbf{O}$ & SHAM baseline & $9.34+1.96$ & $311.33+76.8$ \\
\hline $\mathbf{0}$ & stressed & $28.72+3.66$ & $724.36+126.2$ \\
\hline & CLP baseline & $6.24+2.11$ & $223.60+74.5$ \\
\hline $\mathbf{\Delta}$ & stressed & $16.45+3.5$ & $510.84+141.7$ \\
\hline $\mathbf{\Delta}$ & CLP + DCA baseline & $13.31+2.39$ & $469.27+79.6$ \\
\hline stressed & $16.45+3.5$ & $510.84+141.7$ \\
\hline
\end{tabular}
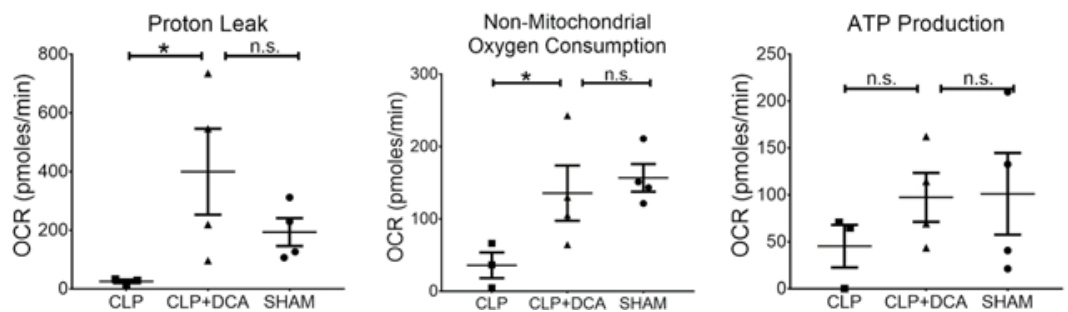

C

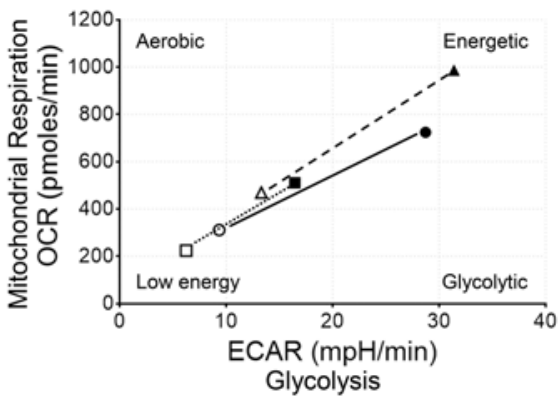
stressed

Figure 2. Dichloroacetate (DCA) effects on isolated hepatocyte mitochondrial bioenergetics. Mitochondrial bioenergetics at 6 hours after DCA or vehicle administered 24 hours after cecal ligation and puncture (CLP) compared with SHAM were assessed using Seahorse XF24. A shows Seahorse tracing averaging oxygen consumption rate (OCR) values from SHAM, CLP (plus vehicle; denoted as CLP), and CLP+ DCA groups. B displays bar graphs of basal and maximum respiration, spare respiratory capacity, proton leak, nonmitochondrial oxygen consumption, and ATP production. Isolated hepatocytes from the CLP+ DCA group showed significantly increased basal and maximum respiration, spare respiratory capacity, proton leak, and nonmitochondrial oxygen consumption compared with CLP, and they were not significantly different compared with SHAM counterparts. Similarly, the energy index in CLP+ DCA group was well above that of CLP alone and SHAM groups (C). $n=4$ for CLP+ DCA treatment, $n=3$ for CLP, $n=4$ for SHAM. Results were assessed by 1 -way ANOVA and Sidak's post hoc test. ${ }^{*} P<0.05 ;{ }^{* *} P<0.01$. Extracellular acidification rate, ECAR.

of OCR for oxidative energy and ECAR as putative glycolytic energy using Agilent software WAVE. Figure 2A shows Seahorse tracing averaging OCR values from SHAM and CLP and CLP ${ }^{+}$DCA groups. Figure 2B displays bar graphs of basal and maximum respiration, SRC, proton leak, nonmitochondrial oxygen consumption, and ATP production. Isolated hepatocytes from mice with DCA (CLP DCA) showed significantly increased basal and maximum respiration, SRC, proton leak, and nonmitochondrial oxygen consumption compared with CLP alone (without DCA), and they were not significantly different from SHAM counterparts. Similarly, energy index in the CLP DCA group was well above that of CLP alone and SHAM groups (Figure 2C).

Splenocytes were also studied from SHAM, CLP, and CLP+ DCA groups; DCA was administered at 24 hours after CLP, and splenocytes were studied 6 hours after DCA (30-hour CLP).

Shown in Figure 3A tracing and Figure 3B bar graphs, in a pattern similar to the isolated hepatocytes, splenocytes from $\mathrm{CLP}^{+}$DCA mice showed a strong trend toward increased basal OCR, maximum respiration, SRC, proton leak, nonmitochondrial oxygen consumption, and ATP production vs. CLP alone and SHAM, although not a statistically significant change. Shown in Figure 3C, we observed that, similar to the isolated hepatocytes, energy index in splenocytes of the CLP ${ }^{+}$DCA group was well above that of CLP alone and SHAM groups.

$D C A$ treatment improves systemic metabolic alterations detectable in serum. We then determined whether the systemic effects of PDC activation by DCA can be detected in serum. Similar to in vivo tolerance, we treated mice with either vehicle or DCA 24 hours after CLP and studied systemic effects (serum bicarbonate 

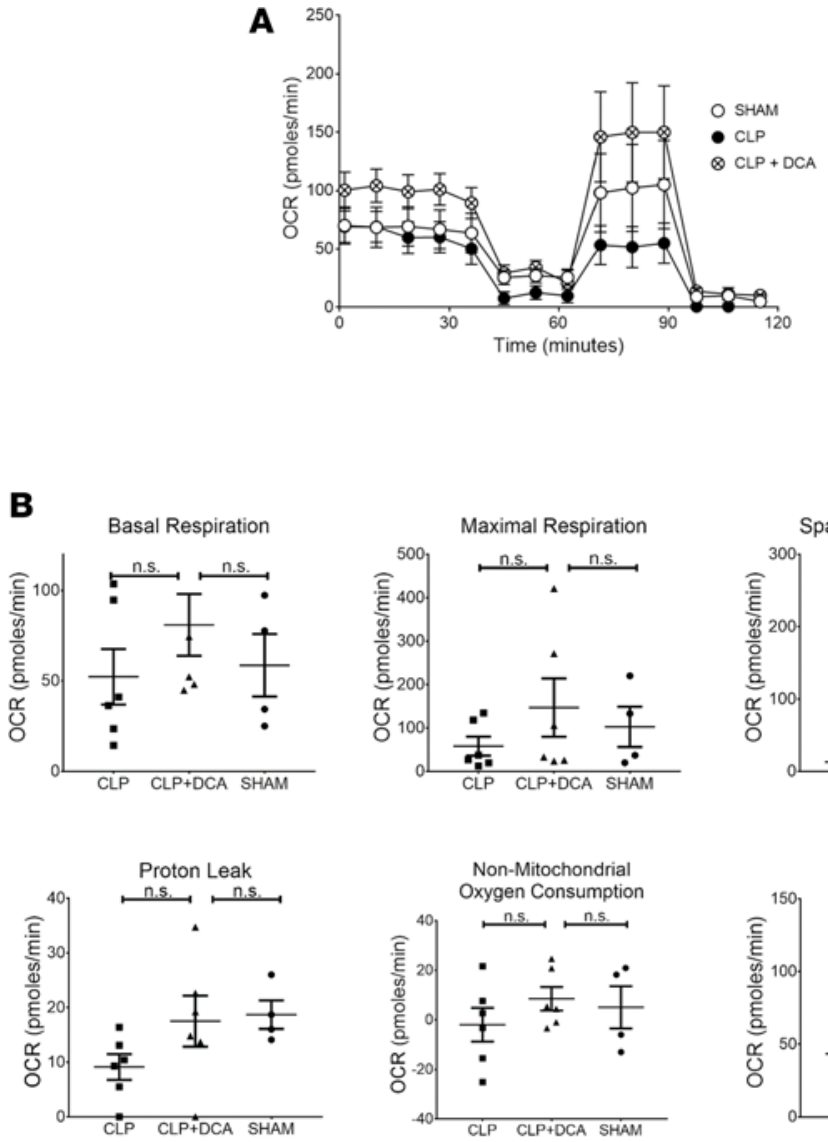

C

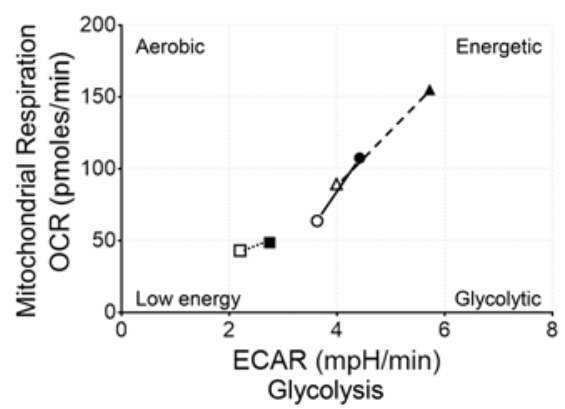

Figure 3. Dichloroacetate (DCA) treatment increases mitochondrial respiration and improves the metabolic potential of mitochondria in splenocytes from septic mice. Seahorse XF24 Analyzer and the mitochondrial stress test was used to determine oxygen consumption rate (OCR) in splenocytes from SHAM $(n=4)$, cecal ligation and puncture (CLP) $(n=6)$, or CLP+ DCA $(n=6)$ mice. A shows tracing of average values from SHAM, CLP, and CLP+ DCA groups. (B) OCR data generated using the mitochondrial stress test by Seahorse WAVE 2.4 Software (Agilent) was analyzed using GraphPad Prism 7.04 Software and ANOVA with Fisher's LSD post-test. (C) The Seahorse WAVE 2.4 Software was used to evaluate the metabolic potential of mitochondria using the extracellular acidification rate (ECAR) and OCR in splenocytes from SHAM $(n=4), \operatorname{CLP}(n=6)$, and DCA-treated CLP mice $(n=6)$ as measured by the Seahorse XFe24 Analyzer.

levels, blood glucose levels, circulating lymphocyte count, and liver enzymes) of DCA 6 hours after treatment (30-hour CLP) described below.

We found that mice with $\mathrm{CLP}^{+}$vehicle developed metabolic acidosis, as indicated by significantly decreased serum bicarbonate levels (Figure 4A). In contrast, mice with CLP ${ }^{+}$DCA treatment showed significantly increased serum bicarbonate levels vs. vehicle-treated septic mice. Because sepsis can cause severe hypoglycemia in mice (19), we measured serum glucose in septic mice treated with vehicle or DCA 24 hours after CLP and followed for 6 hours, before harvesting serum. DCA partially but significantly reversed sepsis-induced hypoglycemia (Figure 4B).

The total circulating lymphocyte count shows promise as a clinically useful biomarker of immune suppression in sepsis and of mortality from continued infection $(20,21)$. Accordingly, we tested this emerging biomarker of immune dysfunction and clinical outcome as a marker of DCA activity. We found that total number of blood lymphocytes was markedly decreased in $\mathrm{CLP}^{+}$vehicle, and DCA administration in sepsis mice (CLP ${ }^{+}$DCA) significantly increased the blood lymphocyte count (Figure 4C).

Sepsis increases metabolic enzyme markers of hepatocellular metabolic dysfunction (22). Accordingly, we tested DCA effects on serum levels of alkaline phosphatase (ALP), aminotransferases (ALT; L-alanine to $\alpha$ ketoglutarate), and aspartate to glutamate (AST). All 3 enzyme biomarkers of hepatic function increased significantly in $\mathrm{CLP}^{+}$vehicle vs. SHAM, and DCA treatment $\left(\mathrm{CLP}^{+} \mathrm{DCA}\right)$ reversed all 3 enzymes to near SHAM levels (Figures 4, D-F). 
A
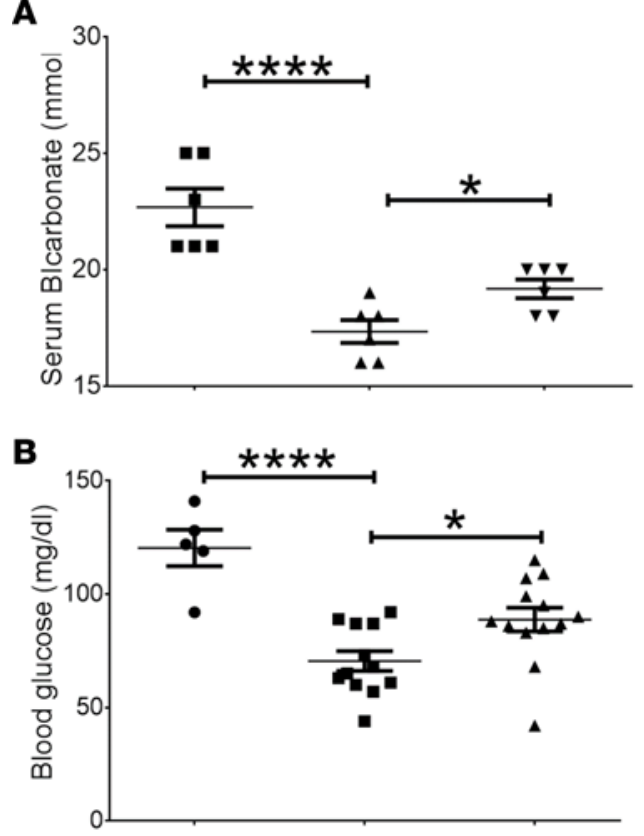

C

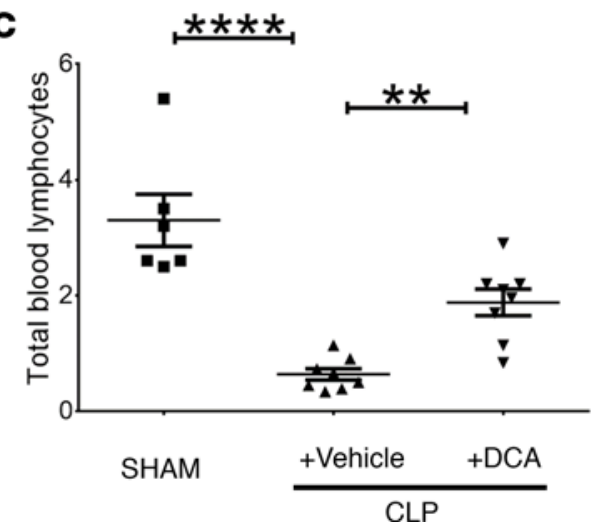

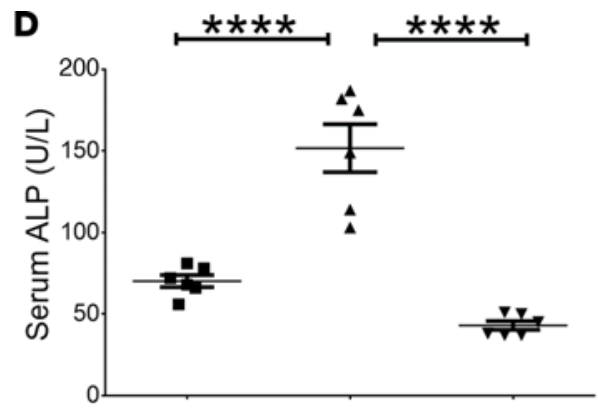
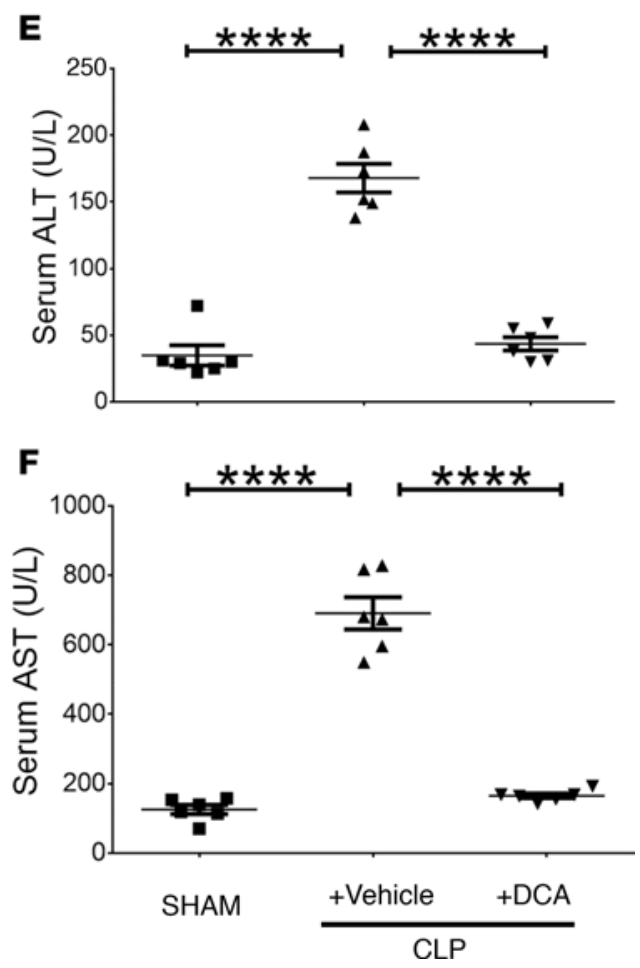

Figure 4. Dichloroacetate (DCA) treatment alters serum and cell markers associated with sepsis. (A) Serum bicarbonate levels in mice with cecal ligation and puncture (CLP) with vehicle were lower than those in SHAM-operated mice; bicarbonate levels significantly increased in $\mathrm{CLP}^{+}$DCA vs. $\mathrm{CLP}^{+}$vehicle group. B shows that serum glucose levels were significantly lower in $\mathrm{CLP}^{+}$vehicle vs. SHAM group and increased in CLP+ DCA vs. CLP+ ${ }^{+}$vehicle mice. $C$ shows a significant decrease in total lymphocyte count in $\mathrm{CLP}^{+}$vehicle vs. SHAM group, with a significant increase in total lymphocyte count in CLP+ DCA vs. $\mathrm{CLP}^{+}$vehicle. $\mathbf{D}-\mathbf{F}$ show that liver enzymes, alkaline phosphatase (ALP) (D), alanine aminotransferase (ALT) (E), and aspartate aminotransferase (AST) (F) increased significantly in CLP+ vehicle vs. SHAM groups and that all 3 enzymes normalized (to SHAM levels) in response to DCA treatment in CLP $^{+}$ DCA vs. CLP+ vehicle group. A, D, E, and F, $n=6$; $\mathbf{B}, n=5$ SHAM, $n=12$ CLP, $n$ $=13 \mathrm{CLP}^{+}$DCA; C, $n=6 /$ SHAM, $n=8 /$ group in $\mathrm{CLP}^{+}$vehicle and CLP+ DCA. Assessed by 1-way ANOVA with Sidak's post hoc multiple comparisons test. ${ }^{*} P$ $<0.05$; ${ }^{* *} P<0.01 ;{ }^{* * *} P<0.0001$.

DCA treatment improves hemodynamics and the microvascular inflammatory response. We next investigated the effects of DCA treatment on hemodynamics and the microvascular-tissue interface (MVI). DCA-treated mice (CLP+ DCA) had significantly higher mean arterial blood pressures compared with vehicle-treated septic animals (Figure 5A). To determine the effect of DCA on the MVI in sepsis, we measured leukocyte and platelet adhesion. Previously, we reported that the MVI develops endotoxin tolerance in vivo within 18 hours of CLP and lasts at least up to 36 hours after CLP (9).Here, to determine the effect of DCA on the endotoxin tolerance in MVI in sepsis, we treated mice with either vehicle or DCA at 24 hours after CLP. We then measured leukocyte and platelet adhesion with and without LPS stimulation as a "second hit" at 6 hours after treatment (30 hours after CLP). We showed that mice $\mathrm{CLP}^{+}$vehicle remained endotoxin tolerant, as published before (Figure 5B) (9). In contrast, in the $\mathrm{CLP}^{+}$DCA group, both leukocyte and platelet adhesion significantly increased in response to LPS administration, indicating that DCA reverses repression of microvascular adhesion; microvascular adhesion is a major innate immune and inflammatory response required for delivery of immune cells to local sites of infection or throughout the circulation. Moreover, these data suggest that the PDC/ PDK axis participates in regulating microvascular homeostasis. We further observed that reversal of endotoxin tolerance by DCA correlated with increased expression of the rate-limiting adhesion molecules ICAM1 and E-selectin in $\mathrm{CLP}^{+} \mathrm{DCA}$ vs. $\mathrm{CLP}^{+}$vehicle group (Figure 5C), indicating a response of endothelial cells to DCA treatment. Expression of these mediators is supported by pathways that require $\mathrm{NF}-\kappa \mathrm{B}$ p65 transcription activation, consistent with the notion that substrate selection drives sepsis bioenergetics reprogramming and controls leukocyte-endothelial adherence interactions. 
A

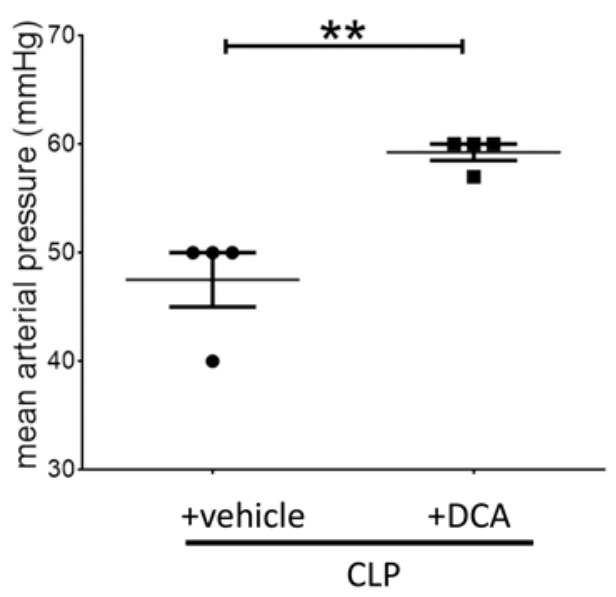

C

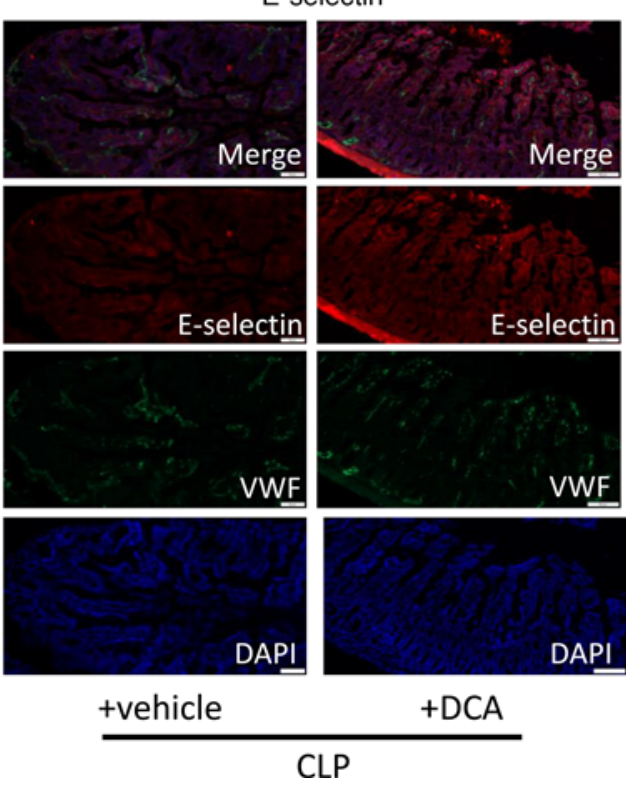

B
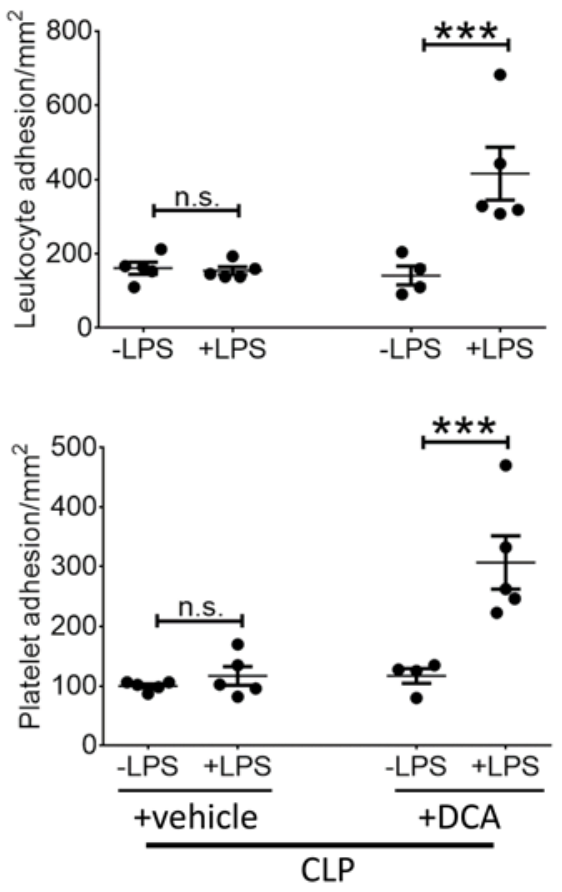

ICAM-1
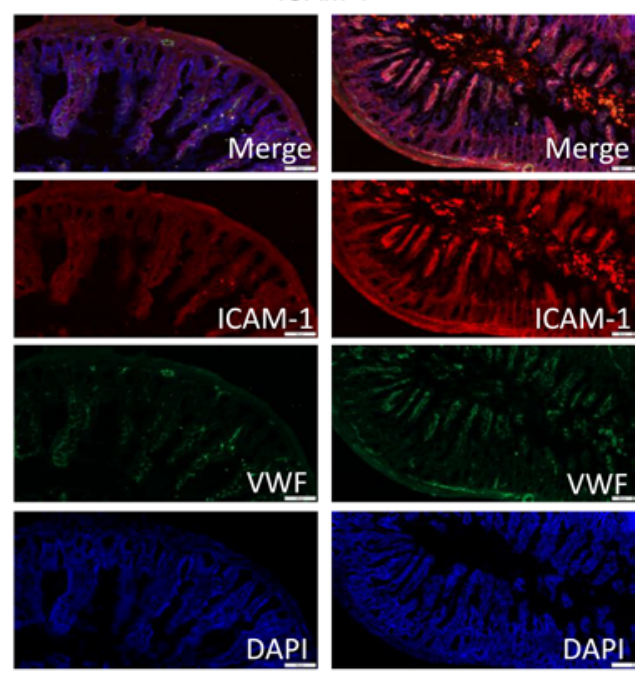

+vehicle + DCA

CLP

Figure 5. Dichloroacetate (DCA) treatment increases blood pressure and improves microvascular dysfunction. Septic animals were treated with DCA or vehicle 24 hours after cecal ligation and puncture (CLP) and assessed 6 hours after treatment. (A) Mean arterial pressure (MAP), measured via carotid artery cannulation, was significantly higher in CLP+ DCA vs. $\mathrm{CLP}^{+}$vehicle group. (B) Mice treated with DCA (CLP+ DCA) or vehicle $\left(\mathrm{CLP}^{+}\right.$vehicle) were challenged with either normal saline (denoted as -LPS) or LPS (+LPS) i.p., and leukocyte and platelet adhesion were determined 4 hours later in the small intestine using intravital microscopy. $\mathrm{CLP}^{+}$vehicle group did not show significant increase in leukocyte or platelet adhesion in response to LPS (+LPS) vs. normal saline (-LPS) stimulation and remained endotoxin tolerant. However, in CLP+ DCA groups, leukocyte and platelet adhesion increased significantly in response to LPS (vs. respective -LPS group), demonstrating endotoxin responsiveness. (C) E-selectin (left panel), ICAM1 (right panel) adhesion molecules, von Willebrand factor (VWF) endothelial marker, and nuclear DAPI staining show qualitatively increased E-selectin and ICAM1 expression in small intestinal tissue sections of $\mathrm{CLP}^{+}$DCA vs. CLP ${ }^{+}$ vehicle group. IHC scale bar: 100 $\mu \mathrm{m} ; \mathbf{A}, n=4$ per group, $t$ test. B, $n$ $=5$ in \pm LPS vehicle and + LPS DCA $n=4$ in -LPS DCA. Assessed by 1-way ANOVA with Tukey post hoc multiple comparison test. C, $n=3$. One exemplary result is shown. ${ }^{*} P$ $<0.01{ }^{* * *} P<0.001$.

$D C A$ repolarizes effector and repressor immune cell polarity in $C D 4^{+} T$ cells and $C D 11 c^{+} D C s$ in septic mice. The metabolic switch from glucose fueling of an effector immune cell resistance phenotype to an immune tolerance phenotype contributes to severe immune suppression and continued infection during animal and human sepsis To examine the effect of PDK inhibition by DCA on this critically important feature of sepsis, we first used flow cytometry to analyze isolated splenocytes for $\mathrm{CD}^{+} \mathrm{T}$ cells and $\mathrm{CD} 11 \mathrm{c}^{+} \mathrm{DC}$ obtained from vehicle-treated and DCA-treated septic mice. Isolated splenocytes were assayed 6 hours after treatment in CLP ${ }^{+}$ DCA vs. $\mathrm{CLP}^{+}$vehicle groups and compared with SHAM control. The proportion of total CD4 ${ }^{+}$cells among total splenocytes decreased during sepsis and was unaffected by DCA (Figure 6A). In contrast, the proportion of $\mathrm{CD}_{2} 5^{+} \mathrm{FoxP}^{+} \mathrm{CD}^{+}$Tregs increased in $\mathrm{CLP}^{+}$vehicle mice (23) (Figure 6B). DCA treatment reversed this increased Treg proportion in $\mathrm{CLP}^{+} \mathrm{DCA}$, supporting a polarity shift to effector CD4 ${ }^{+} \mathrm{Th} 1$ cells (24). Sepsis also increased the frequency of $\mathrm{CD}_{11 \mathrm{c}^{+}}$cells in $\mathrm{CLP}^{+}$vehicle vs. SHAM, which was not appreciably altered in the 

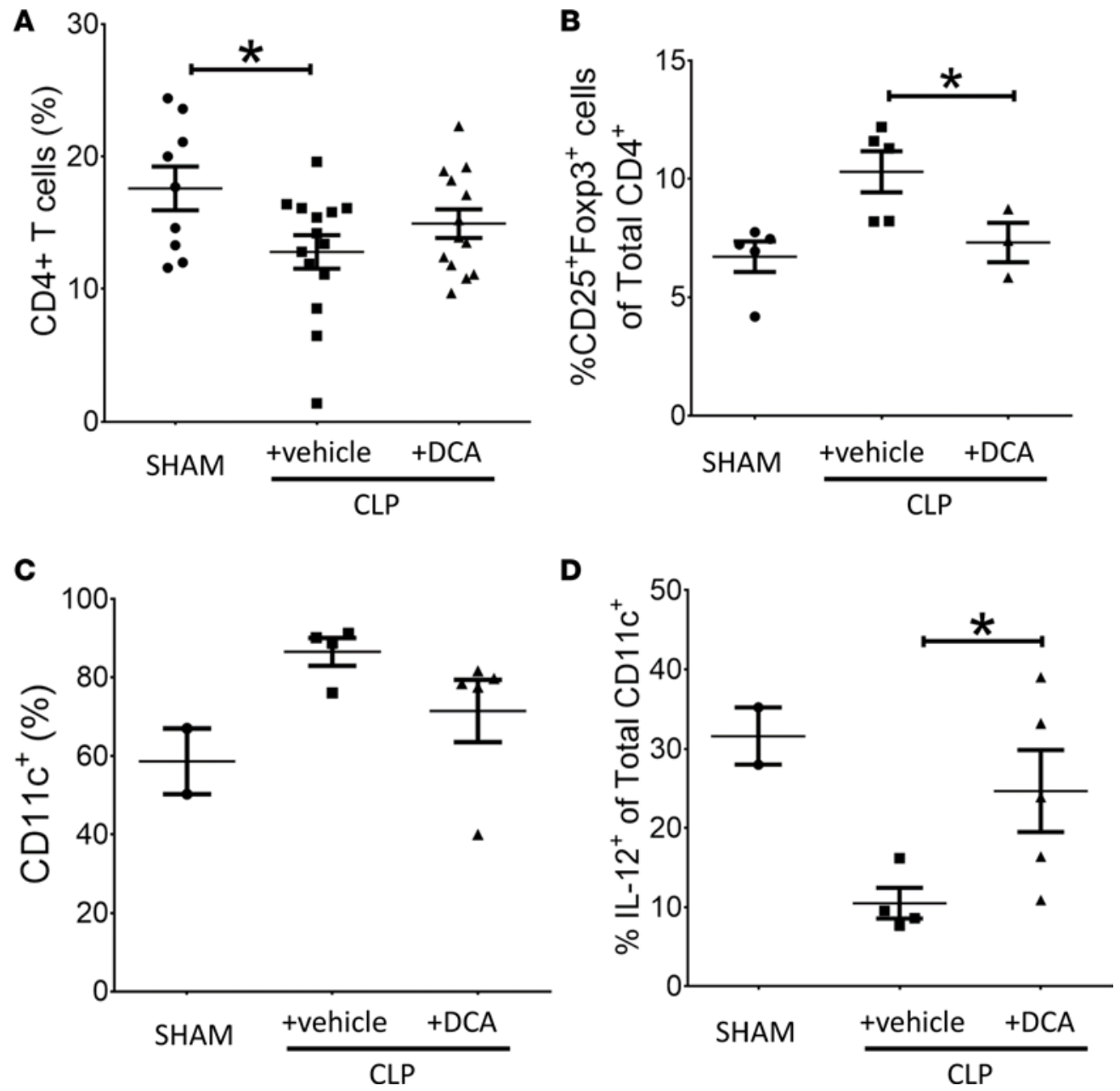

Figure 6. Dichloroacetate (DCA) treatment repolarizes effector and repressor immune cell responses in the spleen. To examine the effect of pyruvate dehydrogenase kinase (PDK) inhibition on immune polarity, we used flow cytometry to analyze isolated splenocytes for $C D 4^{+} T$ cells and $C D 11 c^{+} D C s$ obtained from post-cecal ligation and puncture (CLP) mice with or vehicle (CLP+ vehicle) vs. DCA (CLP+ DCA) treatment. Isolated splenocytes were assayed 6 hours after DCA/vehicle treatments and compared with SHAM control. The proportion of total CD4+ cells among total splenocytes was significantly lower in both the sepsis groups (CLP+ vehicle and CLP+ DCA) and was unaffected by DCA (A). The proportion of $\mathrm{CD} 25^{+} \mathrm{FoxP3}^{+} \mathrm{CD} 4^{+}$Tregs were not significantly higher in $\mathrm{CLP}^{+}$vehicle group vs. SHAM, while the proportion of $\mathrm{CD}_{25}{ }^{+} \mathrm{FoxP3}^{+} \mathrm{CD}^{+}$cells in the CLP+ $\mathrm{DCA}$ group was significantly lower vs. CLP+ vehicle group (B). Sepsis also induced an increase in the frequency of CD11 $\mathrm{C}^{+}$cells in $\mathrm{CLP}^{+}$vehicle vs. SHAM group, and this trend was not affected by DCA (C). However, sepsis caused a marked fall in IL-12+CD11 ${ }^{+}$cell frequency in $\mathrm{CLP}^{+}$vehicle vs. SHAM group and a significant increase in IL-12+CD11 c $^{+}$cells vs. CLP+ vehicle group (D). A, SHAM $n=9$, CLP $n=14, \mathrm{CLP}^{+}$DCA $n=13$; B, SHAM $n=5$, CLP $n=5$, CLP $^{+}$DCA $n=3$; C, SHAM $n=2$, CLP $n=4$, CLP $^{+}$DCA $n=5$; D, SHAM $n=2$, CLP $n=4$, CLP $^{+}$DCA $n=5$. Assessed by 1-way ANOVA with Sidak's post hoc multiple comparisons. ${ }^{*} P<0.05$.

$\mathrm{CLP}^{+}$DCA group (Figure 6C). In contrast, sepsis caused a marked decrease in $\mathrm{IL}-12^{+} \mathrm{CD} 11 \mathrm{c}^{+}$cell frequency in $\mathrm{CLP}^{+}$vehicle vs. SHAM, an index of tolerogenic DCs (2), which was partially reversed by DCA in the $\mathrm{CLP}^{+}$DCA group (Figure 6D). To further analyze immune polarity, we plotted the ratios of immune effector to immune tolerance/repressor cells. Table 1 shows a significant change in the ratio of $\mathrm{CD} 25^{+} \mathrm{FoxP} 3^{+} \mathrm{CD} 4{ }^{+}$ repressor cells to total $\mathrm{CD} 4^{+}\left(\mathrm{CD} 25^{+} \mathrm{FoxP}^{+}: \mathrm{CD} 4^{+}\right)$cells following DCA or vehicle treatment.

$D C A$ reverses immune repressor and activator cytokines in the spleen of septic mice. To further assess the effect of PDK inhibition on immune polarity in the spleen, we defined the IFN- $\gamma$ proimmune and TGF $\beta$ and IL-10 antiimmune axes in $\mathrm{CD}^{-}$and $\mathrm{CD}^{+} \mathrm{T}$ cells. DCA increased the percentage of IFN- $\gamma^{+}$cells of both $\mathrm{CD}^{-}$and $\mathrm{CD} 4^{+} \mathrm{T}$ cells (Figures 7, A and B), but it did not change TGF $\beta$ and IL-10 frequency in CD4- ${ }^{-}$ cells (Figure 7, C and E). However, DCA reversed the sepsis-induced increase in the percentage of TGF $\beta^{+}$ and IL- $10^{+}$cytokines in CD4 $4^{+} \mathrm{T}$ cells (Figures 7, D and F). We then calculated the effect of DCA on the ratios of the immune repressor IL-10 and TGF $\beta$ and immune enhancer (IFN- $\gamma$ ) in splenic cells from septic 
Table 1. Dichloroacetate (DCA) changes the ratios of CD25+FoxP3+ to CD4+ (CD25+ FoxP3': CD4+) cells in cecal ligation and puncture (CLP) mice CLP+ DCA vs. CLP+ vehicle group

\begin{tabular}{|c|c|c|c|}
\hline \hline CLP & +vehicle average ( \pm SEM) & +DCA average ( \pm SEM) & P value $(\boldsymbol{t}$ test) \\
\hline CD25 ${ }^{+}$Fox3p $\mathrm{p}^{+}$CD4total & $0.61( \pm 0.4)$ & $0.37( \pm 0.6)$ & 0.016 \\
\hline
\end{tabular}

animals (Table 2), and we found a significant polarity shift at 6 hours. Together, these data are consistent with the concept that reactivating PDC by inactivating PDK reverses the immune tolerance phenotype in $\mathrm{CD} 4^{+} \mathrm{T}$ cells toward the immune-competent state.

$D C A$ improves small intestinal villus structure and increases $\beta$-catenin, a putative biomarker of cell and organ regeneration. We first examined the effects of DCA on the structural integrity of the small intestine, a sentinel tissue of organ failure in sepsis that is rapidly injured by inflammatory shock states $(25,26)$. We evaluated small intestinal tissue sections by histology. We observed that the blunting of small intestinal villi previously reported in sepsis (27) appeared markedly decreased by DCA (Figure 8A). Our descriptive qualitative structural finding suggested that DCA treatment might rejuvenate dysfunctional organs, as suggested by our serum markers of liver injury.

$\beta$-Catenin promotes organ regeneration and innate and adaptive immune cell differentiation $(28,29)$, and it can be detected by IHC and immunoblots. We found that DCA treatment (CLP DCA) increased $\beta$-catenin staining by IHC compared with vehicle-treated $\left(\mathrm{CLP}^{+}\right.$vehicle-treated) septic mice in the small intestine (Figure 8B), liver (Figure 8C), and kidney tissue in the area of the renal tubules (Figure 8D). In addition, we applied immunoblotting of IHC heart and kidney tissues (Figure 8E). Both kidney and heart (Figure 8E) tissue staining suggested that levels of $\beta$-catenin decreased below basal (SHAM) levels 30 hours after sepsis onset $\left(\mathrm{CLP}^{+}\right.$vehicle) and increased in DCA-treated mice (CLP $\left.{ }^{+} \mathrm{DCA}\right)$, consistent with the IHC results in the organs shown in Figure 8, B-D. To further probe $\beta$-catenin as a possible biomarker of DCA beneficial effects, we used THP-1 human monocytes in a cell culture model of sepsis (30) and found that DCA increased $\beta$-catenin expression in LPS-tolerant cells (Figure 8E). Together, these findings suggest that DCA broadly increases $\beta$-catenin expression, which may be a useful biomarker for cell and organ regeneration.

DCA accelerates bacterial clearance and improves survival in septic mice. Finally, we examined the effects of DCA on survival in murine sepsis. DCA was administered as a single $25 \mathrm{mg} / \mathrm{kg}$ dose given i.p. 24 hours after sepsis induction. We chose this time point because of our previous study that used pharmacological inhibition of SIRT1 to improve survival in murine sepsis (9). We tracked survival by Kaplan-Meyer analysis for 14 days in 4 different cohorts of mice $(n=20 ; 10$ in each of 2 cohorts). The results illustrated in Figure 9A demonstrate that cumulative survival of sepsis animals not receiving DCA treatment $\left(\mathrm{CLP}^{+}\right.$ vehicle) was only $19 \%$ over the 14 -day observation period. In striking contrast, cumulative survival in the DCA-treated animals was $71 \%$. Similar results were achieved when the same dose of DCA was administered by i.v. injection with two $25 \mathrm{mg} / \mathrm{kg}$ doses 12 hours apart (data not shown).

To gain insight into a possible effect of DCA in promoting resolution of sepsis in a model devoid of antibiotic treatment, we determined whether DCA altered bacterial clearance from the peritoneal cavity, the original site of infection. Figure 9B summarizes data from 2 separate cohort experiments and reveals that DCA administration significantly reduced the bacterial content in the peritoneum. Because of the unexpected rapidity of this effect ( 6 hours after DCA dosing and 24 hours after sepsis onset), we determined whether DCA has direct antimicrobial activity by adding or not adding DCA to samples of peritoneal fluid collected from septic mice 30 hours after sepsis. We found no evidence of an antimicrobial effect of the drug (data not shown).

\section{Discussion}

Over 15 million deaths worldwide are due sepsis (31), with 90-day mortality rates for patients in septic shock approaching 50\% (32). Sepsis incurs the greatest in-hospital cost of any illness and is responsible for most deaths in critical care units (33). Clearly, there are serious gaps in understanding this syndrome. This study's major finding is that the PDC/PDK axis plays a critical role in determining sepsis resolution and survival in mice and is a druggable target. PDC stimulation by the small molecule pyruvate analogue 
A

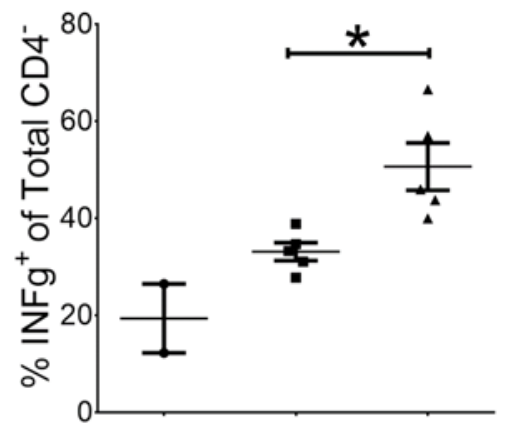

C

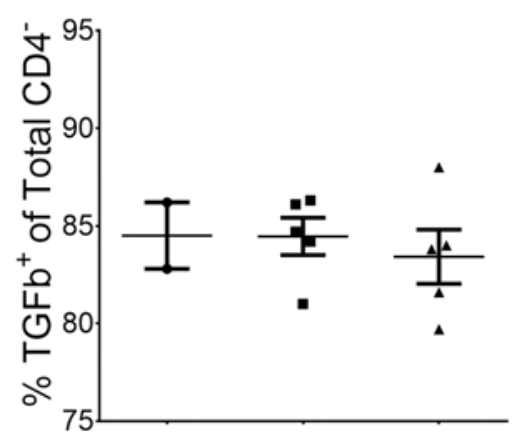

E

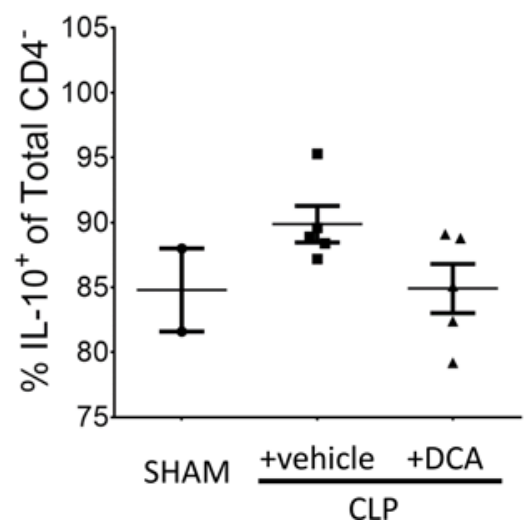

B

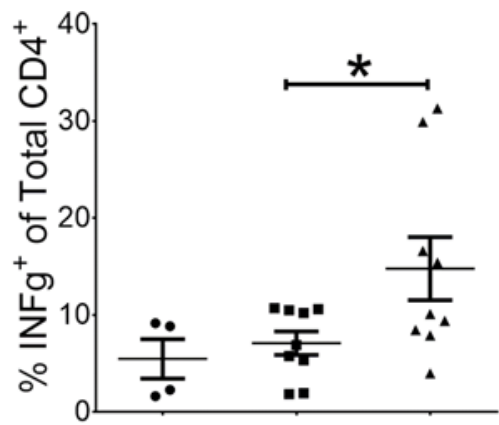

D

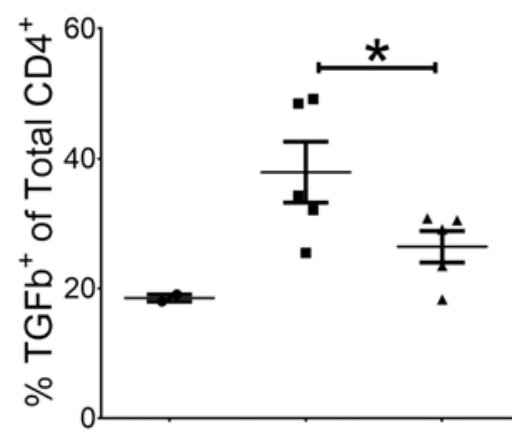

$\mathbf{F}$

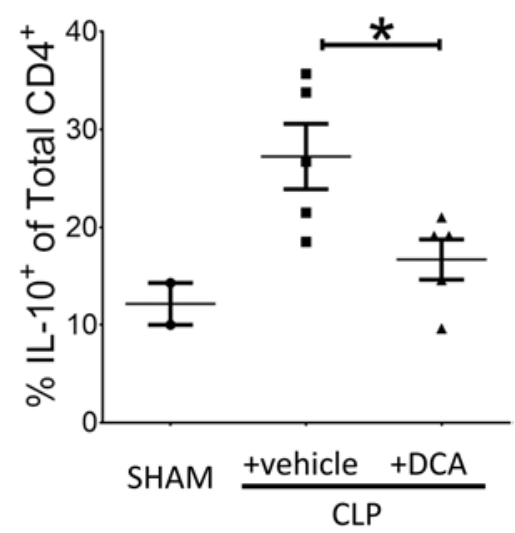

Figure 7. Dichloroacetate (DCA) reverses immune repressor and activator cytokines in the spleen of septic mice. To investigate the effect of pyruvate dehydrogenase kinase (PDK) inhibition on repressor vs. activator cytokines, we assessed the IFN- $\gamma$ (proimmune), TCF $\beta$ (antiimmune), and IL-10 (antiimmune) axes in $\mathrm{CD}^{-}$and $\mathrm{CD} 4^{+} \mathrm{T}$ cells. DCA increased the percentage of IFN- $\gamma^{+}$ cells in both CD4 ${ }^{-}$and in CD4 ${ }^{+}$T cells ( $A$ and B). In contrast, there were no significant difference between $\mathrm{CLP}^{+}$vehicle vs. $\mathrm{CLP}^{+}$ DCA groups in TCF $\beta^{+}$and IL-10+ frequency in CD4 $^{-}$T cells ( $C$ and $\mathbf{E}$ ). DCA reversed the sepsis-induced increase in the percentage of TGF $\beta^{+}$and IL-10+ $10^{+}$cytokines in CD4 $4^{+} \mathrm{T}$ cells (D and F). A, SHAM $n=4$, CLP $n=9$, CLP $^{+}$ DCA $n=9$; Sidak's multiple comparisons. B, $\operatorname{SHAM} n=2, \operatorname{CLP} n=5, \operatorname{CLP}^{+}$DCA $n=5$; Sidak's multiple comparisons. C, SHAM $n=$ 2 , $\mathrm{CLP} n=5, \mathrm{CLP}^{+} \mathrm{DCA} n=5$; Sidak's multiple comparisons. D, SHAM $n=4, \operatorname{CLP} n=9$, $\mathrm{CLP}^{+} \mathrm{DCA} n=9$; Sidak's multiple comparisons. $\mathrm{E}, \mathrm{SHAM} n=2, \operatorname{CLP} n=5, \mathrm{CLP}+\mathrm{DCA} n$ $=5$; Sidak's multiple comparisons. F, SHAM $n=2, \operatorname{CLP} n=5, \mathrm{CLP}^{+}$DCA $n=5$; Sidak's multiple comparisons.

DCA physiologically closes a metabolic gap between the low catabolic energy supply associated with immune tolerance and dysregulated organ recovery and the high energy-consuming anabolic processes required by effector immune cells and organs to resist infection and restore organ function. The notion that mitochondrial oxidative metabolism was enhanced by DCA is supported by improvements in mitochondrial respiration and the cell energy index in isolated hepatocytes and, similarly, a trend toward enhanced mitochondrial respiration and an increased energy index in total splenocytes. The physiologic effects of DCA were rapid, systemic, and potent, as evidenced by increased serum levels of bicarbonate, decreased hypoglycemia, improved blood pressure and microvascular inflammatory responses, reversal of repressed innate and adaptive immunity, and reduced infection burden, all of which improved within 6 hours of a single drug dose delivered 24 hours after sepsis onset in mice. These broad effects of DCA data are consistent with the known pharmacological properties of the small molecule, which has high bioavailability in vivo and stimulates PDC activity in diverse tissues within minutes of its oral or parenteral administration (15). The decisive clinical benefit of the drug in this mouse model was reflected in a marked increase in survival.

Actions of DCA that could be critically important in sepsis include repolarization of splenic CD4 ${ }^{+}$ $\mathrm{T}$ cells and DC11 $\mathrm{c}^{+} \mathrm{DCs}$, which occurred concomitantly with increased levels of the proinflammatory, anabolism-supporting cytokines IFN- $\gamma$ and IL-12 and with decreased concentrations of IL-10. That DCA may, in part, act through cell and organ regenerative processes is supported by our finding 
Table 2. Dichloroacetate (DCA) significantly decreases TGF $\beta$ : IFN- $\gamma$ and TGF $\beta$ : IL-10 ratios in the spleen vs. vehicle-treated CD4 ${ }^{+}$and CD4- cells from mice with cecal ligation and puncture (CLP)

\begin{tabular}{lccc}
\hline CLP & +vehicle average $( \pm$ SEM) & +DCA average $( \pm$ SEM) & P value $(\boldsymbol{t}$ test) \\
CD4- TGF $\beta: I F N-\gamma$ & $2.6( \pm 0.1)$ & $1.7( \pm 0.1)$ & 0.002 \\
CD4- IL-10:IFN- $\gamma$ & $2.8( \pm 0.2)$ & $1.7( \pm 0.2)$ & 0.002 \\
CD4 $4^{+}$TGF $\beta: I F N-\gamma$ & $5.5( \pm 1.0)$ & $1.8( \pm 0.5)$ & 0.03 \\
CD4 IL-10:IFN- $\gamma$ & $4.0( \pm 1.0)$ & $1.2( \pm 0.3)$ & 0.02
\end{tabular}

of increased $\beta$-catenin expression, a well-known contributor of immune and organ regeneration (28), in multiple tissues and cells following drug administration. Another potential biomarker of effects of DCA on immune competence was the reversal of absolute lymphopenia, a predictor of sepsis outcome (21). DCA also improved peritoneal bacterial clearance without the benefit of antibiotics, as reflective of generalized immune enhancement. DCA is a structural analog of the natural PDK inhibitor pyruvate and competes with pyruvate for its binding pocket in PDK (13). By inhibiting PDK, DCA maintains PDC in its unphosphorylated, active state, leading to stimulation of mitochondrial glucose oxidation and the electron chain proton force that increases OXPHOS and ATP production. DCA has been used for decades as an investigational drug for numerous congenital and acquired disorders of mitochondrial metabolism, including diabetes, ischemia-reperfusion injury, coronary revascularization, pulmonary arterial hypertension, and cancer (i.e., conditions in which the efficient mitochondrial conversion of carbohydrate into energy is perturbed; ref. 16). Earlier studies in which DCA was used to treat extremely high levels of lactate in adults, some of whom had sepsis, showed no clinical improvement in survival, despite beneficial effects on acid-base metabolism; one could speculate that it is difficult to show definitive clinical benefit in a heterogeneous population (34-36). More recently, beneficial effects of DCA have been reported in experimental hemorrhagic shock (37), in patients with primary pulmonary arterial hypertension (38), in experimental pulmonary fibrosis (39), in experimental vascular restenosis (40), and in a murine model of cardiac arrest (41), attributable largely or solely to its action on the PDC/PDK axis. To this remarkable pharmacological profile can now be added the present findings of improved immunometabolic function, end-organ restoration, and survival in experimental sepsis, further emphasizing the role of the PDC/PDK axis as a key homeostat in regulating cellular fuel metabolism and function.

Consistent with this notion is mounting evidence for mitochondria as signaling organelles (42-44). Accordingly, the PDC/PDK axis may proximally direct downstream cell functions and diverse reprogramming pathways by increasing availability of acetyl CoA for acetylation reactions in mitochondria, cytosol, and nucleus (45). TCA cycle anabolic products include succinate, fumarate, and aspartate (46). Moreover, promoting hydrogen peroxide generation from superoxide signals proteins by direct cysteine oxidation supports mitochondrial-based ROS signaling pathways (42). SIRT1 and SIRT6, which direct the glycolysis to fatty acid oxidation transition during sepsis, are redox-sensitive proteins, and their deacetylase activities can be reciprocally enhanced or suppressed by reduction or oxidation of specific cysteine thiols $(47,48)$. PDK also is regulated by reversible cysteine oxidation (49). DCA may act through one or more of these signaling pathways to influence the PDC/PDK axis, in addition to its classical inhibitory effect on PDK. We did not identify a specific mechanism by which DCA might inform homeostasis, but the systemic and multifaceted effects of sepsis likely perturb multiple mechanisms that couple to metabolism and bioenergetics, the leading example being innate and adaptive immune epigenetic and posttranslational reprogramming (1).

Finally, and relevant to this study, we recently discovered that delayed and increased mitochondrial SIRT4 gene expression, by an unknown feedback path to the nucleus, represses PDK expression, stimulates PDC decarboxylation to acetyl CoA, increases glycolysis and glucose oxidation, and increases the mitochondrial energy index in monocytes, while breaking immune tolerance (50). Accordingly, mitochondrial-located SIRT4 is a physiologic regulator of acute inflammatory resolution and immunometabolic homeostasis, which — at least in part — inhibits PDK deactivation and restores pyruvate-dependent bioenergetics similar to DCA in this study. 
A
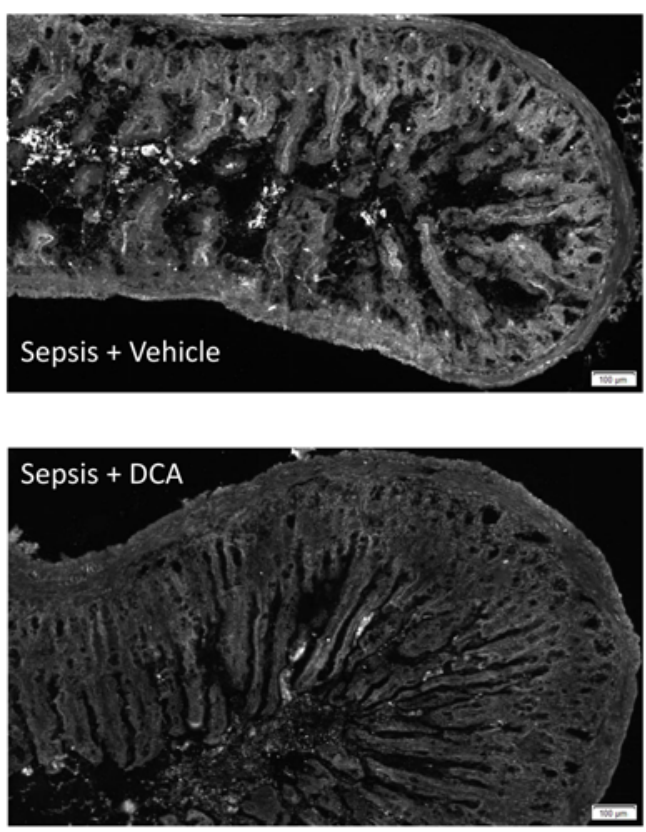

D
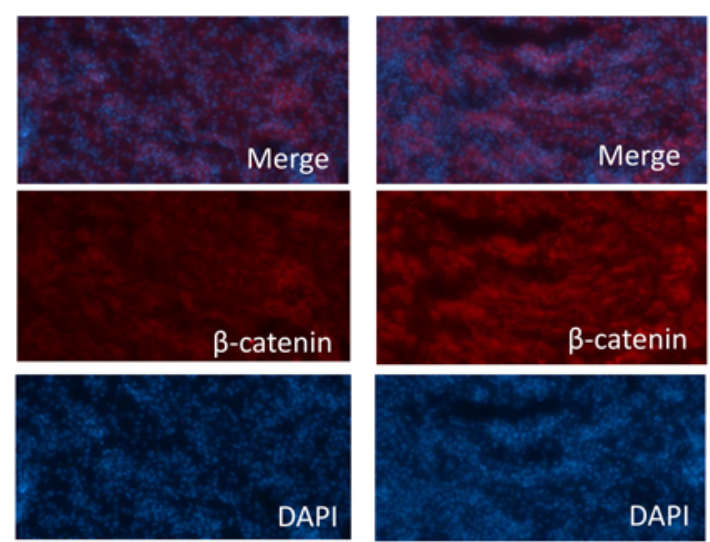

E

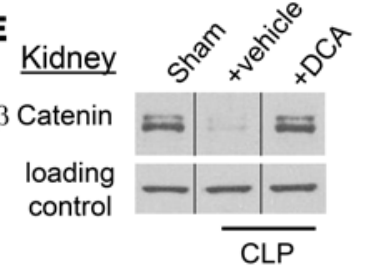

Heart

$\beta$ Catenin

loading

control

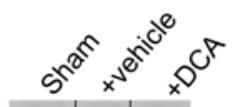

C
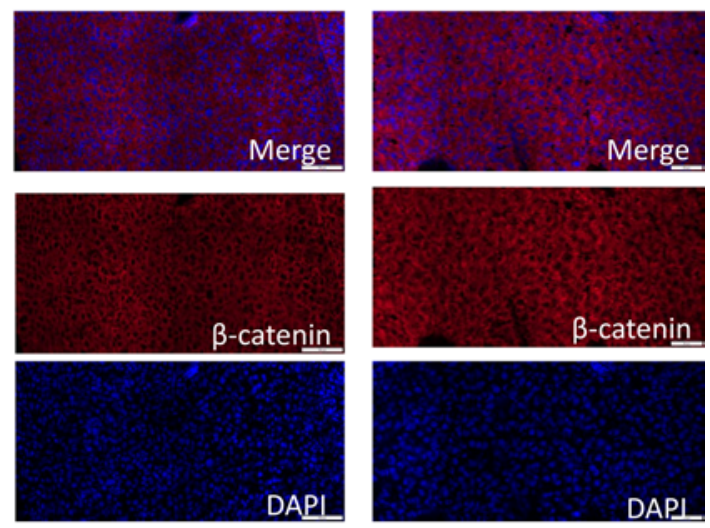

Figure 8. Dichloroacetate (DCA) improves small intestinal villus structure and increases expression of the $\beta$-catenin anabolic transcription factor for cell and organ regeneration pathways. (A) Small intestine sample from mice with cecal ligation and puncture (CLP) with vehicle or DCA were examined by light microscopy and assessed in a blinded fashion. Blunting of small intestinal villi occurred as expected after 30 hours of sepsis. CLP+ DCA mice showed improved structural features of the intestine, with a possible increase in villus length, vs. CLP $^{+}$vehicle group (A). To assess effects of DCA on organs and cells, we used organ and immune cell regeneration promoter $\beta$-catenin and compared $\operatorname{CLP}^{+}$vehicle vs. CLP+ DCA treatment using IHC. $\beta$-Catenin immunofluorescence increased $\mathrm{CLP}^{+} \mathrm{DCA}$ compared with $\mathrm{CLP}^{+}$vehicle group in small intestine (B), liver (C), and kidney (D) tissue sections. To further examine this potential anabolic marker, we performed immunoblots in different tissues and cells in SHAM, CLP vehicle, and CLP ${ }^{+}$ DCA groups. $\beta$-Catenin expression decreased in $\mathrm{CLP}^{+}$vehicle vs. SHAM in kidney and heart tissue samples (E). We also found in a human monocyte in vitro cell model of sepsis 24 hours after endotoxin tolerance development that $5 \mathrm{mM} \mathrm{DCA}$ increased $\beta$-catenin expression (E). IHC scale bar: $100 \mu \mathrm{m} . n=3$ animals were studied in each cohort for each tissue using IHC. For immunoblots, $n \geq 2$ experiments, and 1 exemplary experiment is shown for isolated hepatocytes, heart, and kidney tissue each. For THP-1 cells, 3 distinct in vitro experiments were performed, and 1 example is shown.

In summary, this study provides strong support for the role of the PDC/PDK axis in regulating mitochondrial bioenergetic pathways needed to rejuvenate immunity and organ function during life-threatening sepsis and supports that harnessing glucose oxidation by DCA promotes immunometabolic and organ homeostasis and survival. Finally, the potential efficacy of small molecule intracellular 

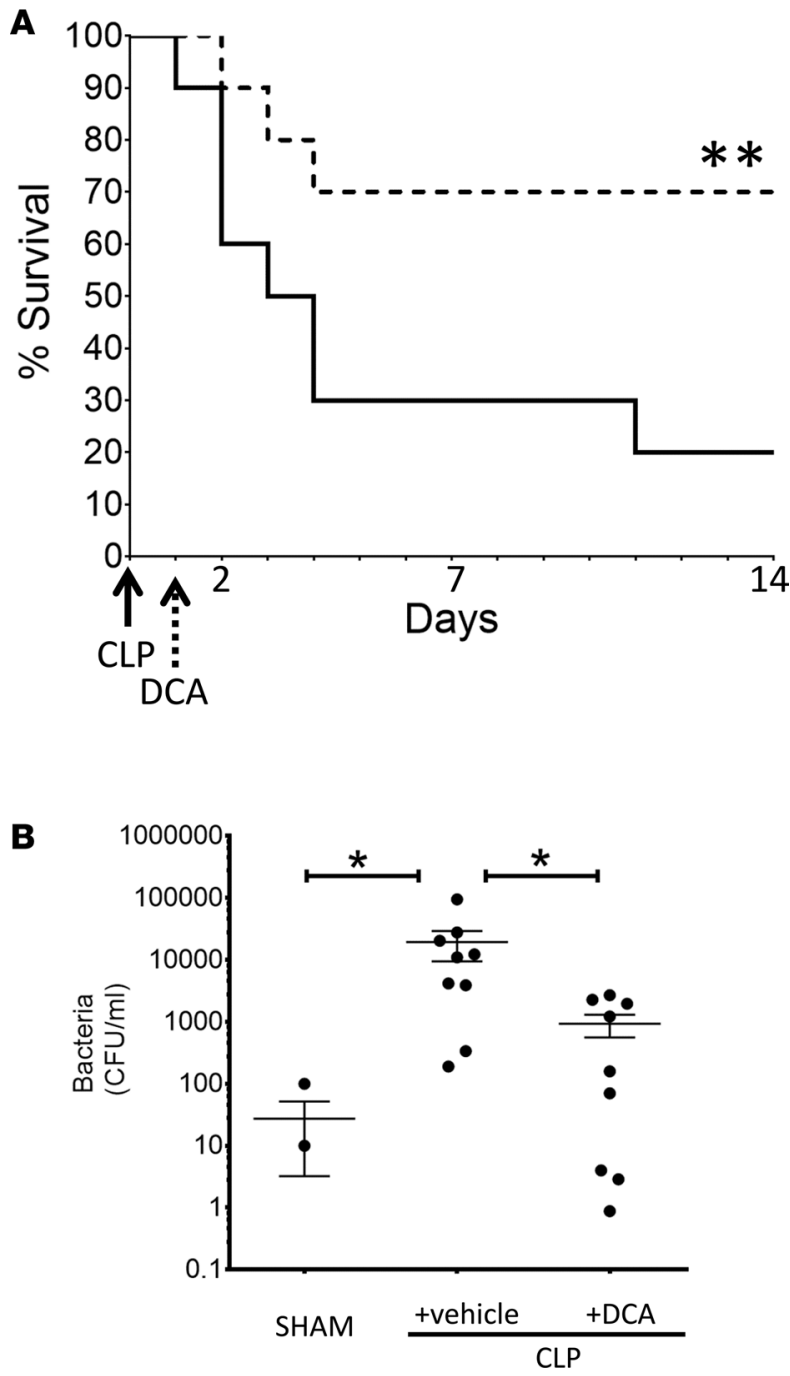

Figure 9. Dichloroacetate (DCA) improves survival and accelerates bacterial clearance in septic mice. (A) To assess the effect of DCA on survival, we treated mice 24 hours after cecal ligation and puncture (CLP) with a single i.p. dose of $25 \mathrm{mg} / \mathrm{kg}$ of DCA (CLP+ DCA) vs. a vehicle control (CLP+ vehicle) and followed 14-day survival. Kaplan-Maier survival curve shows that DCA (CLP+ DCA) significantly improved 14-day survival vs. vehicle treatment $\left(\mathrm{CLP}^{+}\right.$vehicle) in the absence of antibiotics. $n=20$ in each of 2 cohorts. $n=$ 20 mice/cohort; Log-rank (Mantel-Cox) test. ${ }^{* *} P<0.01$. (B) To determine the effect of infection in the absence of antibiotics, we assessed bacterial clearance from the peritoneal cavity, using colony count methodology. Animals were treated with vehicle or DCA as a single dose of $25 \mathrm{mg} / \mathrm{kg}$ i.p. 24 hours after CLP sepsis induction, and samples were obtained 6 hours after vehicle or DCA treatment. DCA significantly reduced the microbial colony counts in the peritoneum. SHAM, $n=$ 4; CLP, $n=7$; CLP ${ }^{+}$DCA, $n=7$,. Sidak's multiple comparisons test. Note: Since the axis is logarithmic, only values greater than zero can be plotted. For this graph, 2 values were zero or negative, so they are not visible on the graph.

physiologic effects on mitochondrial bioenergetics shown in this study opens a new door for better understanding and treating dysregulated inflammation associated with sepsis.

\section{Methods}

\section{Murine model of sepsis}

All mice (C57B1/6 male mice; 6-8 weeks) were purchased from the Jackson Laboratory and maintained in an AAALAC-approved animal resources facility at Wake Forest School of Medicine. We used the CLP sepsis model in C57Bl/ 6 mice with 40\%-50\% survival in which no antibiotics were administered (9).

\section{DCA treatment}

A single i.p. dose of DCA as a sodium salt (MilliporeSigma) $25 \mathrm{mg} / \mathrm{kg}(4 \mathrm{ml} / \mathrm{kg})(51)$ or PBS control (vehicle) were given at 24 hours, at which time microvascular inflammation has transitioned to an endotoxin tolerant state (8). For tissue, hepatocyte isolation, or splenocyte isolation, mice were euthanized at 6 hours after DCA/vehicle treatment (30 hours after CLP) using isoflurane anesthesia and cervical dislocation (as a secondary method). Splenocyte and hepatocyte isolation are discussed below.

\section{Microvascular inflammation}

Two hours after DCA/vehicle treatment, we used intravital microscopy to evaluate the effect of $E$. coli LPS O111:B4 (LPS, $5 \mu \mathrm{g} / \mathrm{mouse}$ i.p., 4 hours of stimulation) or normal saline to assess delineate leukocyte and platelet adhesion interactions within the small intestinal microcirculation (9). 


\section{Splenocyte and hepatocyte isolation}

Splenocyte isolation. Splenocytes were isolated from mice from SHAM, CLP ${ }^{+}$DCA, and CLP ${ }^{+}$vehicle treatment groups as described above. After euthanasia, the abdomen was opened and the spleen was retrieved and mechanically disrupted. Cells were isolated by passing the homogenized material in sterile PBS through a 70- $\mu \mathrm{m}$ nylon screen. Splenocytes were pelleted and then treated with ACK to lyse RBC; they were then washed 3 times with sterile PBS. After the last wash, splenocytes were pelleted, resuspended in sterile PBS, and counted. Isolated cells $\left(3 \times 10^{5} /\right.$ well) were plated in 24-well plates for the Seahorse XF measurement.

Hepatocyte isolation. Hepatocytes were isolated using previously described methods (52). Briefly, mice were anesthetized, and the portal vein was cannulated using a 25-gauge needle; then, the inferior vena cava was cut. For SHAM mice, the liver was perfused at $5 \mathrm{ml} / \mathrm{min}$ with $1 \times$ HBSS containing $0.5 \mathrm{mM}$ EDTA and $10 \mathrm{mM}$ HEPES for 7 minutes, followed by $0.025 \%$ collagenase solution containing $5 \mathrm{mM} \mathrm{CaCl} 2$ for 7 minutes. For CLP mice, the initial perfusion was done at $4 \mathrm{ml} / \mathrm{min}$ for 8 minutes, followed by $0.05 \%$ collagenase solution containing $5 \mathrm{mM} \mathrm{CaCl} 2$ for 5 minutes. The liver was removed, and hepatocytes were isolated by mincing the liver into small pieces in William's E medium containing 1\% L-glutamine and 1\% penicillin/streptomycin and then filtering through a $100-\mu \mathrm{m}$ cell strainer. Viability in the final preparations was $80 \%-90 \%$. Cells were plated in $10 \%$ FBS containing medium at a density of $1 \times 10^{6}$ cells per well either on collagen-coated or uncoated 6-well plates for treatments. All treated cells were then transferred to 24-well plates for Seahorse XF 24 measurements.

\section{Mitochondrial respiration and bioenergetics}

Mitochondrial respiration and bioenergetics were studied using Seahorse XF 24 (Seahorse Inc.). Cells were fed with medium containing nutrient concentrations as follows: $100 \mathrm{mM}$ Pyruvate, $200 \mathrm{mM}$ Glutamine, 2.5 M Glucose. We used a mitochondrial stress test to measure OCR; ECAR indirectly measured extracellular lactic acid secretion rates. OCR were analyzed for (a) basal mitochondrial respiration; (b) ATP production and uncoupling (after adding oligomycin Complex V ATPase inhibitor); (c) respiratory reserve (after completely uncoupling respiration with FCCP); and (d) maximal respiration, assessed by blocking the electron transfer chain (ETC) with rotenone (Complex I) and antimycin (Complex III). An energy index, which plots the ratio of OCR to ECAR, was calculated using WAVE software. We used isolated hepatocytes and splenocytes to assess sepsis and DCA treatment effects on bioenergetics; isolation procedures are described above.

IHC

Small intestinal and liver tissue acetone fixed frozen sections of tissue were stained by IHC as reported (9). Antibodies included E-selectin (catalog SC14011; Santa Cruz Biotechnology Inc.), ICAM1 (catalog BD550287; BD biosciences), Von Willebrand factor (VWF) (catalog AB11717, Abcam), and $\beta$-catenin (catalog 9587; Cell Signaling Technologies). Virtual images were captured as described previously (9).

\section{Flow cytometry}

Antibodies used included anti-mouse CD25-PerCP-Cy5.5 (catalog 45-0251), anti-mouse CD3e-APCeFluor780 (catalog 47-0031), anti-mouse CD4-PE-Cy7 (catalog 25-0041), anti-mouse CD45-PE (catalog 25-0041), anti-mouse CD8a-FITC (catalog 11-0081), and anti-mouse Foxp3-eFluor450 (catalog 48-5773; all purchased from eBioscience). Stained cells and compensation controls were analyzed using a FACS CantoII (BD Biosciences) and FowJo vv10.0.4 software (Treestar Inc.) (9).

\section{Bacterial clearance}

Mice were treated without or with DCA. Trypticase soy agar with $5 \%$ sheep blood plates were allowed to come to room temperature while the frozen peritoneal fluid samples thawed. The spread plate method was used to plate sample (neat) volumes between 10 and $500 \mu 1$ and a $10 \mu 1$ volume of a $10 \times$ dilution. Plates sat at room temperature until fluid was absorbed, before inverting the plate and incubating at $37^{\circ} \mathrm{C}$. After 24 hours, plates were counted, and counts between 30 and 300 were used to calculate CFUs per ml.

\section{Serum analyses}

These assays were performed by IDEXX Laboratories Inc., a company that specializes in monitoring biochemical and pathological changes in laboratory animals. 


\section{Quantitative PCR}

mRNA was quantified as described (2). Data were normalized to GAPDH mRNA and presented as fold change relative to mRNA of untreated cells.

\section{Western blots}

Levels of mouse PDK1 and mouse PDC E1 $\alpha$ in 30-hour SHAM, CLP ${ }^{+}$vehicle, and CLP+ DCA mice (stated above) (9). Primary antibodies anti-rabbit PDK1 (Enzo Life Sciences, catalog ADI-KAPPK112-D), anti-rabbit pyruvate dehydrogenase pSer232 and anti-rabbit PDC pSer300 (Calbiochem, catalogs AP1063 and AP1064, respectively) were used following manufacture's instruction. Protein levels were quantified using Image J software. THP-1 cells used for Western blot analysis of $\beta$-catenin were purchased from ATCC.

\section{Statistics}

Data were analyzed using Graph Pad Prism 6.0. Analyses with more than 3 samples were analyzed using 1-way ANOVA with Sidak's using GrapPad Prism or Tukey's post hoc multiple comparisons using Statview (SAS). Two-way student $t$ tests were used where appropriate. $P<0.05$ was designated as significant. Log rank test was used to compare survival between the groups in the Kaplan-Meier survival curves, and $P<0.05$ was designated as significant. In the figures, ${ }^{*} P \leq 0.05 ;{ }^{* *} P \leq 0.01 ;{ }^{* * *} P \leq 0.001 ;{ }^{* * * *} P \leq 0.0001$. All data presented as mean \pm SEM.

\section{Study approval}

The IACUC of the Wake Forest School of Medicine approved all the experiments. All the animal experiments were performed according to the NIH guidelines. All the mice were monitored at least twice daily for pain and distress, and humane end points were strictly followed.

\section{Author contributions}

CEM and VV jointly directed the study and wrote the manuscript. TL and MZ conducted the mouse metabolic studies and had conceptual input into the project. AM conducted the studies on immunity and small bowel. PWS guided DCA studies and interpretations and helped write the manuscript. DLL conducted the cell-based studies of metabolic deactivation. MZ measured and analyzed PDHK1 and PDH assays and studied the in vitro monocyte deactivation model. NLB acted as lead director of animal surgery and pathology. RJWA and MN helped design and interpret results and provided study consultation. BKY advised on methods, interpreted data, designed the figures and legends, and edited the manuscript.

\section{Acknowledgments}

This work was supported by NIH grants R01AI079144, R01AI065791 and S1, and R01GM102497 to CEM; and GMR01099807 to VV. European Research Council (ERC) Consolidator Grant (no. 310372) supported MGN, and GM099871, FD005407, HD089804 supported PWS. The authors thank Manish Baharadwaj and the Wake Forest University Bioenergy Core Laboratory under direction of Anthony Molina for supporting the metabolic analyses using Seahorse XF24 respirator and Young Choi for technical assistance.

Address correspondence to: Charles. E. McCall or Vidula Vachharajani, Bowman Gray Center, 575 Patterson Ave, Winston-Salem, NC 27101, USA. Phone: 336.713.4259; Email: chmccall@wakehealth.edu (C.E. McCall). Phone: 336.716.4498; Email: Vidula.Vachharajani@wakehealth.edu (V. Vachharajani).

1. Norata GD, et al. The Cellular and Molecular Basis of Translational Immunometabolism. Immunity. 2015;43(3):421-434.

2. O'Neill LA, Pearce EJ. Immunometabolism governs dendritic cell and macrophage function. J Exp Med. 2016;213(1):15-23.

3. Olenchock BA, Rathmell JC, Vander Heiden MG. Biochemical Underpinnings of Immune Cell Metabolic Phenotypes. Immunity. 2017;46(5):703-713.

4. O'Sullivan D, Pearce EL. Immunology. Expanding the role of metabolism in T cells. Science. 2015;348(6238):976-977.

5. Singer M. The role of mitochondrial dysfunction in sepsis-induced multi-organ failure. Virulence. 2014;5(1):66-72.

6. Singer M. Cellular dysfunction in sepsis. Clin Chest Med. 2008;29(4):655-660.

7. Liu TF, Vachharajani VT, Yoza BK, McCall CE. NAD+-dependent sirtuin 1 and 6 proteins coordinate a switch from glucose to fatty acid oxidation during the acute inflammatory response. J Biol Chem. 2012;287(31):25758-25769.

8. Liu TF, Vachharajani V, Millet P, Bharadwaj MS, Molina AJ, McCall CE. Sequential actions of SIRT1-RELB-SIRT3 coordinate 
nuclear-mitochondrial communication during immunometabolic adaptation to acute inflammation and sepsis. $J$ Biol Chem. 2015;290(1):396-408.

9. Vachharajani VT, et al. SIRT1 inhibition during the hypoinflammatory phenotype of sepsis enhances immunity and improves outcome. J Leukoc Biol. 2014;96(5):785-796.

10. Cheng SC, et al. Broad defects in the energy metabolism of leukocytes underlie immunoparalysis in sepsis. Nat Immunol. 2016;17(4):406-413.

11. Langley RJ, et al. An integrated clinico-metabolomic model improves prediction of death in sepsis. Sci Transl Med. 2013;5(195):195ra95.

12. Langley RJ, et al. Integrative "omic" analysis of experimental bacteremia identifies a metabolic signature that distinguishes human sepsis from systemic inflammatory response syndromes. Am J Respir Crit Care Med. 2014;190(4):445-455.

13. Stacpoole PW. The pyruvate dehydrogenase complex as a therapeutic target for age-related diseases. Aging Cell. 2012;11(3):371-377.

14. Sutendra G, Michelakis ED. Pyruvate dehydrogenase kinase as a novel therapeutic target in oncology. Front Oncol. 2013;3:38.

15. James MO, Stacpoole PW. Pharmacogenetic considerations with dichloroacetate dosing. Pharmacogenomics. 2016;17(7):743-753

16. Patel KP, O'Brien TW, Subramony SH, Shuster J, Stacpoole PW. The spectrum of pyruvate dehydrogenase complex deficiency: clinical, biochemical and genetic features in 371 patients. Mol Genet Metab. 2012;106(3):385-394.

17. Reynolds LM, et al. Transcriptomic profiles of aging in purified human immune cells. BMC Genomics. 2015;16:333.

18. Brand MD, Nicholls DG. Assessing mitochondrial dysfunction in cells. Biochem J. 2011;435(2):297-312.

19. Saito H, Sherwood ER, Varma TK, Evers BM. Effects of aging on mortality, hypothermia, and cytokine induction in mice with endotoxemia or sepsis. Mech Ageing Dev. 2003;124(10-12):1047-1058.

20. Unsinger J, et al. IL-7 promotes T cell viability, trafficking, and functionality and improves survival in sepsis. J Immunol. 2010;184(7):3768-3779.

21. Drewry AM, Samra N, Skrupky LP, Fuller BM, Compton SM, Hotchkiss RS. Persistent lymphopenia after diagnosis of sepsis predicts mortality. Shock. 2014;42(5):383-391.

22. Hotchkiss RS, Karl IE. The pathophysiology and treatment of sepsis. N Engl J Med. 2003;348(2):138-150.

23. Wei J, Raynor J, Nguyen TL, Chi H. Nutrient and Metabolic Sensing in T Cell Responses. Front Immunol. 2017;8:247.

24. Gerriets VA, et al. Metabolic programming and PDHK1 control CD4+ T cell subsets and inflammation. J Clin Invest. 2015;125(1):194-207.

25. Yoseph BP, et al. Mechanisms of Intestinal Barrier Dysfunction in Sepsis. Shock. 2016;46(1):52-59.

26. Klingensmith NJ, Coopersmith CM. The Gut as the Motor of Multiple Organ Dysfunction in Critical Illness. Crit Care Clin. 2016;32(2):203-212.

27. Williams JM, et al. A mouse model of pathological small intestinal epithelial cell apoptosis and shedding induced by systemic administration of lipopolysaccharide. Dis Model Mech. 2013;6(6):1388-1399.

28. Valenta T, Hausmann G, Basler K. The many faces and functions of $\beta$-catenin. EMBO J. 2012;31(12):2714-2736.

29. $\mathrm{Ke} \mathrm{B}$, et al. $\beta$-catenin regulates innate and adaptive immunity in mouse liver ischemia-reperfusion injury. Hepatology. 2013;57(3):1203-1214

30. LaRue KE, McCall CE. A labile transcriptional repressor modulates endotoxin tolerance. J Exp Med. 1994;180(6):2269-2275.

31. Angus DC. The lingering consequences of sepsis: a hidden public health disaster? JAMA. 2010;304(16):1833-1834.

32. Singer M, et al. The Third International Consensus Definitions for Sepsis and Septic Shock (Sepsis-3). JAMA. 2016;315(8):801-810.

33. Torio CM Moore BJ. National Inpatient Hospital Costs: The Most Expensive Conditions by Payer, 2013: Statistical Brief \#204. In: Healthcare Cost and Utilization Project (HCUP) Statistical Briefs. Rockville, MD; 2006.

34. Stacpoole PW, et al. A controlled clinical trial of dichloroacetate for treatment of lactic acidosis in adults. The Dichloroacetate-Lactic Acidosis Study Group. N Engl J Med. 1992;327(22):1564-1569.

35. Stacpoole PW, Lorenz AC, Thomas RG, Harman EM. Dichloroacetate in the treatment of lactic acidosis. Ann Intern Med. 1988;108(1):58-63.

36. Stacpoole PW, Harman EM, Curry SH, Baumgartner TG, Misbin RI. Treatment of lactic acidosis with dichloroacetate. NEngl JMed. 1983;309(7):390-396.

37. Subramani K, et al. Mitochondrial targeting by dichloroacetate improves outcome following hemorrhagic shock. Sci Rep. 2017;7(1):2671.

38. Michelakis ED, et al. Inhibition of pyruvate dehydrogenase kinase improves pulmonary arterial hypertension in genetically susceptible patients. Sci Transl Med. 2017;9(413):eaao4583.

39. Goodwin J, et al. Targeting Hypoxia-Inducible Factor-1 $\alpha$ /Pyruvate Dehydrogenase Kinase 1 Axis by Dichloroacetate Suppresses Bleomycin-induced Pulmonary Fibrosis. Am J Respir Cell Mol Biol. 2018;58(2):216-231.

40. Deuse T, et al. Dichloroacetate prevents restenosis in preclinical animal models of vessel injury. Nature. 2014;509(7502):641-644.

41. Piao L, Fang YH, Kubler MM, Donnino MW, Sharp WW. Enhanced pyruvate dehydrogenase activity improves cardiac outcomes in a murine model of cardiac arrest. PLoS One. 2017;12(9):e0185046.

42. Reczek CR, Chandel NS. ROS-dependent signal transduction. Curr Opin Cell Biol. 2015;33:8-13.

43. Chandel NS. Mitochondria as signaling organelles. BMC Biol. 2014;12:34.

44. Diebold L, Chandel NS. Mitochondrial ROS regulation of proliferating cells. Free Radic Biol Med. 2016;100:86-93.

45. Menzies KJ, Zhang H, Katsyuba E, Auwerx J. Protein acetylation in metabolism - metabolites and cofactors. Nat Rev Endocrinol. 2016;12(1):43-60.

46. Martínez-Reyes I, et al. TCA Cycle and Mitochondrial Membrane Potential Are Necessary for Diverse Biological Functions Mol Cell. 2016;61(2):199-209.

47. Long D, et al. The Oxidative State of Cysteine Thiol 144 Regulates the SIRT6 Glucose Homeostat. Sci Rep. 2017;7(1):11005.

48. Kornberg MD, et al. GAPDH mediates nitrosylation of nuclear proteins. Nat Cell Biol. 2010;12(11):1094-1100.

49. Hurd TR, et al. Inactivation of pyruvate dehydrogenase kinase 2 by mitochondrial reactive oxygen species. J Biol Chem. 2012;287(42):35153-35160.

50. Tao J, Zhang J, Ling Y, McCall CE, Liu TF. Mitochondrial Sirtuin 4 Resolves Immune Tolerance in Monocytes by Rebalancing 
Glycolysis and Glucose Oxidation Homeostasis. Front Immunol. 2018;9:419.

51. Kato-Weinstein J, Lingohr MK, Orner GA, Thrall BD, Bull RJ. Effects of dichloroacetate on glycogen metabolism in B6C3F1 mice. Toxicology. 1998;130(2-3):141-154.

52. Li WC, Ralphs KL, Tosh D. Isolation and culture of adult mouse hepatocytes. Methods Mol Biol. 2010;633:185-196. 